Meta

Journal des traducteurs

Translators' Journal

\title{
Humbles mots, mots de prestige et mots de soufre
}

\section{Pierre Agron}

Volume 18, numéro 1-2, mars 1973

Actes du deuxième colloque international de linguistique et de traduction. Montréal, 4-7 octobre 1972

URI : https://id.erudit.org/iderudit/001859ar

DOI : https://doi.org/10.7202/001859ar

Aller au sommaire du numéro

Éditeur(s)

Les Presses de l'Université de Montréal

ISSN

0026-0452 (imprimé)

1492-1421 (numérique)

Découvrir la revue

Citer cet article

Agron, P. (1973). Humbles mots, mots de prestige et mots de soufre. Meta, 18(1-2), 189-200. https://doi.org/10.7202/001859ar d'utilisation que vous pouvez consulter en ligne.

https://apropos.erudit.org/fr/usagers/politique-dutilisation/ 


\section{Humbles mots, mots de prestige et mots de soufre}

On est toujours un peu surpris chaque fois que l'on constate combien de gens instruits - mais qui n'ont jamais prêté une attention spéciale à leur langage - ignorent qu'un mot, en général, a plusieurs significations. Lorsqu'ils s'en aperçoivent, ce sont des lamentations. Comme tout serait simple, disent-ils, si à chaque mot ne correspondait qu'une notion; à chaque notion un seul mot.

Ils rejoignent ainsi le petit groupe de personnes — bien averties, elles - qui rêvent d'une langue, idéale à leurs yeux, qui satisferait notamment à la condition ci-dessus. \& Ce comportement ne se manifeste pas avec intensité en chaque occasion ; il n'en est pas moins général, et explique, au moins en partie, l'adoption sans contrôle des termes étrangers. \

Comme un langage n'est pas un objet fabriqué une fois pour toutes mais qu'il dépend de la société humaine qui en use, nous allons examiner comment en pratique se passent les choses, pour quelle catégorie de mots le nombre des acceptions se réduit, spontanément pour ainsi dire, pour quelle autre il augmente, et comment il est possible d'intervenir.

À l'aube de la biennale de la langue française de Menton, Jean Darbelnet faisait remarquer que, lorsque par malheur une des acceptions d'un mot devient péjorative, les autres acceptions de ce mot tombent en désuétude, le mot ainsi marqué n'est plus employé que dans cette acception aux vapeurs de soufre. La loi de J. Darbelnet peut être énoncée sous une forme remarquable : «La mauvaise acception chasse les bonnes. » Remarquable, car on y reconnaît une célèbre loi financière : «La mauvaise monnaie chasse la bonne.» Ce qui veut dire que, si dans un pays une monnaie d'or a cours légal en même temps qu'une autre monnaie, de papier par exemple, le papier circule seul, tandis que l'or reste au fond des coffres.

Cette loi financière a été attribuée à plusieurs pères, mais il semble bien que le premier à la formuler ait été un esprit universel du quatorzième siècle, mathématicien, théologien, philosophe, économiste, ministre du roi de France et évêque de la charmante petite ville de Lisieux, Nicole d'Oresme.

Jean Darbelnet se trouve donc en brillante compagnie. $\AA$ Menton il a illustré sa loi par un exemple qui, je pense, a un peu surpris les Français. Au 
Canada, a-t-il dit, le mot «exploitation », à cause de «l'exploitation de l'homme par l'homme », n'est plus employé qu'en liaison avec l'exploiteur ; l'autre acception, liée à exploitant, est morte.

Il me semble qu'en France cette seconde acception est toujours vivante. Et personnellement j'hésite à donner une valeur trop générale à la première. De la parabole de Marthe et de Marie, j'ai retenu que nous sommes tantôt exploiteurs et tantôt exploités, tantôt Marthe et tantôt Marie, les deux ensembles parfois, en proportion variable suivant les idées que nous nous laissons mettre en tête par les propagandistes.

Je vais, à mon tour, donner un exemple qui, je le crains, va paraître puéril aux Canadiens. Lors de la réorganisation de la France à la fỉn du $18^{e}$ siècle, la première assemblée de la première république, reprenant idée et mot proposés vingt-cinq ans plus tôt, remplaça les anciennes provinces par des unités administratives plus petites : les départements, qui reçurent des noms en rapport avec leur situation géographique. Dans les Basses-Alpes les montagnes étaient moins élevées que dans les Hautes-Alpes; le Bas-Rhin était en aval du Haut-Rhin; et la Seine inférieure, etc., à l'embouchure des fleuves du même nom.

La Charente inférieure produit du cognac. Voilà une vingtaine d'années, une association de producteurs se demanda ce qui se passerait si un acheteur du Texas ou de São Paulo s'imaginait que l'adjectif correspondait à la qualité du produit. Vue sous un tel angle, la situation était intolérable. Les pressions exercées sur l'administration durent être suffisamment fortes, car rapidement, malgré l'inertie bien connue des bureaux parisiens, l'adjectif « inférieur » fut remplacé par «maritime » ou « atlantique ». L'esthétique y gagna, le commerce aussi peut-être. Suivant la loi de J. Darbelnet, l'acception de soufre avait chassé l'acception topographique. Le record est aujourd'hui détenu par le département des Basses-Alpes, complètement transformé en Alpes de Haute-Provence.

Les malheureux, dans cette affaire, sont les Bretons, qui restent avec leurs «Côtes du Nord ». C'est un pays célébré dans une des plus belles pages de notre littérature : "Je suis né, déesse aux yeux bleus, [...] chez les Cimmériens bons et vertueux qui habitent au bord de la mer sombre, hérissée de rochers, toujours battue par les orages. On y connaît à peine le soleil [...] Les nuages y paraissent sans couleur, et la joie même y est un peu triste. »

C'est très beau, mais du point de vue touristique, quelle catastrophe, car cela confirme le mot «nord», qui à lui seul fait froid dans le dos et chasse les «vacanciers». Un beau nom de remplacement est tout prêt : "Côtes d'Armor »; l'armor en breton est le pays de la mer, par opposition à l'argoat, pays de la terre. Jusqu'à présent, l'administration résiste : peut-être attend-elle que, par la grâce du code postal, tous ces noms et toutes ces querelles disparaissent dans l'anonymat des nombres?

«Et l'Orne inférieure ?» demanderont les connaisseurs. Ce fut l'objet du premier conflit, il y aura bientôt deux siècles. Les représentants de ce département protestèrent, en effet, devant l'Assemblée constituante : «Pourquoi serions-nous inférieurs aux gens de l'Orne ?»Contresens qui montre l'influence de la mauvaise 
acception. Les assemblées révolutionnaires étaient plus conciliantes qu'on ne pourrait le croire ; on fit droit à la requête : «Quel nom voulez-vous ?» «- Celui des récifs au large de nos côtes. » Et c'est ainsi qu'à peine né, le département de l'Orne inférieure devint le Calvados.

"Qu'est-ce que ça veut dire, Calvados ?» demandions-nous au vieux maître d'école normand, qui nous racontait cette histoire. « - C'est le nom d'un vaisseau de la Grande Armada, que la tempête brisa sur ces récifs, le Salvador. » Longtemps après, j'ai appris que cette étymologie n'est peut-être pas aussi sûre qu'il le croyait, mais comment pourrais-je en changer maintenant sans peiner sa mémoire?

En toute rigueur il faudrait parler d' «acceptions de soufre », mais la loi de Jean Darbelnet permet de parler de «mots de soufre», puisque finalement il ne reste qu'une acception en activité.

Je n'ai rencontré, et cela récemment, qu'un seul écart à cette loi : un conservateur de musée, présentant une exposition de livres rares, écrit : «Nos rois n'ont pas cherché à se procurer systématiquement [...] les productions de l'art livresque considérées aujourd'hui comme chefs-d'œuvre de l'époque.» «Livresque » n'a qu'une acception et elle est péjorative. Ce mot n'avait pas à figurer ici ; il fallait «l'art du livre ». De telles méprises sont rares.

La loi de J. Darbelnet est d'autant mieux observée que l'acception est plus déplaisante. Aussi peut-elle, suivant un mouvement constant en science, être utilisée à rebours, pour juger de la force d'un concept de soufre, de son affaiblissement ou de son renforcement dans le temps. Il y a, en effet, des degrés dans les mots de soufre. Certains sont permanents : tyran, despote... La loi pour eux s'applique à plein. Qui se souvient des sens initiaux ?

D'autres mots n'ont valeur de soufre que pendant un temps ou en un lieu déterminé. À Paris le nom d'Anatole France donné à une partie du quai d'Orsay provoqua une vive réaction de la part des commerçants touchés (antiquaires ou hôteliers). Le nom de "quai d'Orsay » est connu du monde entier, et ils voyaient déjà leur chiffre d'affaires diminué par cet hommage à un écrivain oublié. Voilà pour l'espace.

Voici pour le temps : le mot «collaborateur » désigna une personne suspecte, à tort ou à raison, de sympathie pour l'envahisseur. Il y a trente ans la force de cette acception était telle qu'il n'était plus possible de dire : «Je me félicite du collaborateur que je viens d'embaucher. " Depuis, heureusement l'oubli, nuit sombre...

Le mot «incendie» était un mot «bas » pour $\mathbf{M}^{\text {me }}$ de Sévigné ; il fallait parler d'embrasement. En sens inverse l'évolution des mœurs frappe d'interdit des mots jusqu'alors d'usage courant, leur domaine d'emploi se réduit comme flot qui se retire. Les flaques laissées en arrière sont des expressions figées, d'usage plus ou moins rare, où le mot tabou, suivant l'usage ancien, ne choque pas.

Il est des mots de soufre faibles, qui ne provoquent qu'un malaise, plus ou moins perçu. Les chirurgiens se servent de pinces pour réduire le flux de sang; 
une « clip-cave » est une telle pince appliquée sur la veine cave. L'expression gêne paraît-il. Mais ce qui déplaît ce n'est pas « clip », mot court et vif, c'est « cave »; on pense malgré soi, comme le petit héros des Allumettes suédoises, à un "lieu redoutable où un ignoble fil d'araignée vous colle au visage, où l'odeur de moisi vous prend à la gorge... » Une association de clip avec le nom d'une autre veine, clip-jugulaire, clip-fémorale, n'aurait pas rencontré d'opposition, linguistique tout au moins.

Ce malaise a parfois des conséquences importantes. Les mots «vulgarisation », "vulgarisateur» souffrent de leur parenté avec vulgaire. La mise de la science à la portée d'un grand nombre de gens est, à cause de cette parenté, une activité mésestimée. Les scientifiques français de valeur la pratiquent trop peu. Situation dommageable. Le concept est des plus utiles; il faudrait lui trouver un autre nom. On peut même regretter que « vulgaire » n'ait pas une force de répulsion suffisante pour rendre impossible l'usage de "vulgarisation» dans son acception actuelle et nous contraindre à le remplacer.

A titre d'expérience, et pour un instant seulement, essayons à cette place un mot d'origine voisine : populariser. Le résultat n'est pas remarquable, mais déjà ce mot improvisé fait lever dans l'esprit images et souvenirs moins déplaisants.

L'Association des écrivains scientifiques de France, qui fait des efforts méritoires pour améliorer la qualité de la vulgarisation, devrait bien mettre au concours le remplacement du mot par un de ces noms que, dans un moment, nous appellerons « de prestige ».

Le modèle des mots de soufre devrait être «diable», évidemment. Il n'en est rien. On pouvait entendre récemment à la radio parisienne : « Le diable est plein de bonnes intentions; la preuve, c'est qu'on dit un pauvre diable, on ne dit pas un pauvre dieu!» Je pense que le brillant essayiste, auteur de ces propos, ne doit pas tenir sa preuve pour très probante. Mais il nous rappelle ainsi que le mot «diable \& a perdu beaucoup de sa force. Le manutentionnaire qui pousse un diable n'a pas le sentiment d'user d'un outil diabolique. Diable a plusieurs sens, diabolique un seul. Il a gardé sa force, ainsi que démon ou démoniaque.

Le cas de «démon» est une belle vérification de la loi de J. Darbelnet. Car ce mot, en principe, a deux acceptions. Ceux qui s'adonnent à cette activité passionnante qu'est la physique, font connaissance presque en même temps avec deux petits personnages qu'affectionnent les physiciens, le bonhomme d'Ampère et le démon de Maxwell. Si le premier oriente la circulation dans les champs magnétiques sans faire d'histoires, le démon de Maxwell est autrement inquiétant. Les jeunes étudiants l'imaginent en Méphistophélès, tout de rouge vêtu, longue queue, oreilles pointues; il arrête certaines molécules et en laisse passer d'autres de façon à mettre en défaut la thermodynamique. Or, ce n'était pas l'image que Maxiwell avait en tête quand, pour les besoins de sa cause, il l'avait imaginé. Il pensait à un génie, à un demi-dieu, à un être capable d'accomplir avec aisance des tâches hors des possibilités humaines. 
C'était également l'acception utilisée par le général von Klück à propos de Foch. Les généraux allemands de la Grande Guerre s'ils reconnaissaient volontiers les mérites de Joffre, évitaient par une curieuse censure morale de se prononcer sur Foch. Pressé de questions par l'ambassadeur d'Angleterre, von Klück finit par dire : «Il est difficile de juger le maréchal Foch, car il y a du « daïmon » en lui. Ce qui signifiait que les réactions militaires de Foch étaient inhabituelles, insolites, qu'elles n'avaient pas été prévues dans les écoles de guerre allemandes.

Il est curieux de noter chez un autre stratège allemand un propos équivalent. Seulement, comme Adolf Hitler ne savait pas le grec, le mot «daïmon» lui échappait, et il se borna à qualifier $\mathrm{d}$ '《 imbéciles militaires » les généraux britanniques, qui n'en apprécièrent pas moins l'hommage dont la brutalité garantissait la sincérité.

On fuit les mots de soufre; on recherche les mots de prestige. Lorsqu'une acception est prestigieuse, les autres acceptions du mot bénéficient plus ou moins de ce prestige. On peut donc là encore parler de mots de prestige.

Comment les nommer ces mots? Les proustiens proposeraient «mordoré ». En hommage à Jules Romains on peut aussi penser à « superbes» : ces mots superbes se détachent alors de la masse des mots humbles, dont nous parlerons plus loin. En attendant, regardez-les passer, mordorés, superbes, ces mots de prestige, en un cortège flamboyant à la manière des peintres de la Renaissance, Paolo Ucello ou Benozzo Gozzoli.

En tête on s'attendrait à trouver le mot « dieu». Si l'on ne dit pas, en effet, un pauvre dieu, on dit «beau comme un dieu ». C'est là sans doute un legs de l'aimable paganisme grec. Mais il est des souvenirs d'autres origines et d'une autre nature : «Le cruel dieu des Juifs l'emporte aussi sur toi. \& Les Hébreux avaient si peur de nommer (leur) dieu qu'ils avaient recours à des initiales : Iod, Hé, Vav, Hé, d'où nous avons tiré Jéovah. Cela peut sembler naï, car, initiales ou non, c'est toujours nommer. Mais les Hébreux croyaient que les noms appartenaient aux êtres et que prononcer un nom était déjà agir sur son possesseur. Nous avons, d'ailleurs, bien du mal à nous libérer de cette croyance.

Chaque peuple nous a transmis sa métaphysique, notre vocabulaire en garde trace. Celui qui porte la toge de pourpre, l'imperator des mots de prestige, est sans conteste le mot "génie ». Faut-il, en effet, que son prestige soit solide pour qu'il résiste depuis si longtemps à tant d'abus.

Certes, si nous surprenons quelque ménagère annonçant que son aîné fait son service dans le génie nous n'en serons pas surpris ; nous avons trop l'habitude, en France tout au moins, de ces activités militaires. Mais, ce cas à part, tous les autres usages de ce mot sont colorés par l'acception prestigieuse. Ainsi, il n'y a pas longtemps, apprenant qu'à l'École polytechnique de Montréal existait une section vouée au «génie de l'environnement», j'ai entendu le mot avec une majuscule. Il m'a fallu un petit moment pour réagir. J'ai revécu les discussions d'il y a vingt ans lorsque les expressions "génie atomique », "génie chimique », ont commencé à se répandre. Si la majorité des spécialistes les avaient adoptées 
avec allégresse, des esprits sérieux tentaient un rappel à l'ordre : « Soyons modestes. On va se moquer de nous. » Les uns et les autres sentaient bien que derrière ces noms était le prestige de "génie ».

Ces choix étaient légitimes, les concepts étaient importants et méritaient de tels noms. Malheureusement il est souvent difficile de maintenir ces noms à leur place. Voici, en témoignage, les histoires récentes de «fiable» et du préfixe «cryo ».

Pour « fiable » les choses se sont déroulées à l'envers de la normale. Consultés, les spécialistes du langage auraient dû déclarer : «Très bonne famille, admise aux honneurs de la poésie dès le $12^{\mathrm{e}}$ siècle ; fiablement se trouve dans le Roman de la rose, infiable chez Montaigne. $\gg$ Littré, qui était originaire de la région la plus conservatrice de la Normandie, ne déclare-t-il pas : «Fiable est un archaïsme se survivant en Normandie où il est très employé. »

Nous pourrions ajouter aujourd'hui que si «fiable » a disparu en Normandie, il reste actif au Canada, où l'on dit couramment qu'un homme est fiable. Que les Normands installés au Canada aient légué ce mot à leurs descendants n'est pas surprenant. Ce qui étonne plutôt, soit dit en passant, c'est la différence de comportement à l'égard des notions de confiance et de méfiance. Les Canadiens me semblent attacher à la seconde beaucoup moins d'importance que leurs cousins du vieux pays. «Méfie-té, méfie-té encore, méfie-té toujours» est une règle de vie soigneusement observée de nos jours dans les campagnes normandes.

Si nous avions proposé «fiable» aux physiciens et aux mécaniciens il y a vingt ans, "Vous êtes bien aimables, nous auraient-ils dit, mais que voulez-vous que nous fassions? $\gg \mathrm{Il}$ y a une douzaine d'années la situation s'est inversée. Les extraordinaires progrès des techniques ont mis à la disposition des hommes des dispositifs auxquels on pouvait accorder un degré de confiance naguère inimaginable. Il fallut créer une sorte de superlatif de sûr. "Très sûr » ne suffisait pas ; «très, très sûr », comme on entend souvent, pas davantage.

Lorsque le 21 juillet 1969 les Américains se sont posés sur la Lune, un commentateur a négligemment déclaré : «Tout va bien, il était prévu que le module lunaire en se posant aurait 42 secondes de carburant d'avance, or il a 100 secondes d'avance. » Cette phrase donne une bonne idée de la précision de l'ensemble des dispositifs en jeu. Si l'on en doute, que l'on songe à la tête que feraient les voyageurs d'un simple vol d'une ville à l'autre, si, au moment de poser son appareil, le pilote prononçait à leur intention la phrase ci-dessus.

Les Américains eurent recours à un vieux mot français : « reliable ». Les Français commencèrent à l'utiliser. Rien ne s'y opposait. Le fait que, dans notre langue, "reliable » ait vu au cours des âges son domaine se rétrécir, ne gênait en rien. Il n'y avait aucun risque d'équivoque entre l'art de la reliure et les montages électroniques raffinés.

Mais le paradoxe continua. Les techniciens estimèrent trop marqué le sens français de reliable. Ils avaient tort; ils montraient ainsi leur ignorance des éléments de la linguistique. On put même lire sous d'augustes plumes des décla- 
rations de ce genre : «Le mot anglais reliability est à peu près imprononçable par une bouche française normale. » Mais qui aurait empêché de le prononcer comme le mot français qu'il avait été ?

Enfin, le fait est là : ce sont des techniciens qui allèrent chercher fiable. D'ordinaire la résistance est grande; on pouvait penser que fiable, s'il n'était pas rejeté, ne serait admis que peu à peu. Il n'en fut rien. En quelques mois, le succès de fiable était complet, reliable avait définitivement disparu.

C'est alors que l'attitude des «littéraires » fut surprenante. Ils déclanchèrent contre fiable un tir de barrage particulièrement dense. On l'attaqua au nom de l'esthétique : comme ce mot est laid! On l'attaqua au nom de la simplicité : pédant en diable, ce mot là ! Fiable est dans le Littré ! Oui, mais seulement dans le Supplément. C'est donc du second choix. Fiable est désuet, nous dit-on, pourquoi ne prenez-vous pas « fidible»?

Un grammairien donna le curieux argument suivant : fiable était un mot bien formé lorsque le verbe «fier» existait; mais fier ayant été remplacé par «se fier», fiable est devenu incorrect et ne peut plus être employé. En outre, ajoutait-il, les adjectifs formés d'un radical de verbe et du suffixe «able» ont une valeur passive; ce qui n'est pas le cas de fiable. Il mentionnait, honnêtement, que «valable » faisait exception.

Lorsque l'on propose un néologisme, l'une des conditions les plus importantes, celle dont on doit tenir le plus grand compte, est le maintien de la simplicité. Il ne faut compliquer la langue que si le bénéfice escompté compense largement cette complication. Inscrire «fiable » à la suite de «valable » dans une catégorie grammaticale exceptionnelle est un inconvénient minime, et l'on pouvait estimer que «fiable» rendrait des services justifiant cette addition.

Le record de fantaisie reste la propriété d'un journaliste : «Par la magie de cette science nouvelle, la fiabilité... " C'était là le titre de son article, dans le corps duquel la fiabilité était devenue une technique. Quant au mot lui-même, il le désignait, en confondant signifié et signifiant, comme «un terme d'anglais qui englobe les études et les essais que l'on fait subir à une pièce... » Ultérieurement, il devait préciser que le mot « fiabilité » était la forme française d'un terme américain fabriqué de toutes pièces et qui se trouvait dans tous les dictionnaires anglo-saxons.

La fiabilité n'est ni une science, ni une technique, mais une qualité, comme la fidélité ou la sensibilité (d'un instrument). Peu importait, d'ailleurs, au journaliste, qui ne voulait qu'être l'écho d'une mode. Utilisés à propos d'objets exceptionnels, fiable et fiabilité, aux yeux du grand public, étaient devenus des mots de prestige. Et tout le monde voulait s'en servir. À en croire la publicité, tout ce qui a un moteur, réfrigérateurs, machines à laver, automobiles naturellement, est fiable. Si l'histoire du sous-préfet aux champs, d'Alphonse Daudet, devait être réécrite, nul doute que l'on commencerait par nous faire remarquer - à juste titre d'ailleurs - combien sa calèche ( « cocher devant, laquais derrière ») est fiable! 
Tout cela est bien aimable, mais de pareils abus détruisent la terminologie. Le seul intérêt de «fiable » était d'être un superlatif. Dès l'instant où l'on multiplie ses usages, il se décolore.

Si l'histoire de «cryo» est différente, les conclusions sont les mêmes. Jusqu'à ces dernières années les phénomènes se passant à des températures inférieures à celle de l'azote liquide n'étaient étudiés qu'en de rares laboratoires. On compte maintenant les utiliser dans l'industrie.

Sur l'initiative de Nicolas Kurti, professeur à Oxford, et de grande renommée, il fut décidé de mettre sur pied une terminologie aussi complète que possible. Un groupe international de quelques spécialistes se chargea de cette tâche, le Comité d'étude des termes techniques français prêtant son concours comme conseiller linguistique.

Un principe, dès l'origine, fut proposé par N. Kurti : recourir au grec, à ses préfixes notamment, pour marquer un degré supérieur, les préfixes latins étant laissés au niveau du langage courant. Ainsi la terminologie des utilisations actuelles du froid, tant industrielles que domestiques, reposerait sur le préfixe latin «frigo», tandis que la nouvelle science et ses applications seraient dotées de termes basés sur le préfixe grec « cryo ».

Quelques mauvaises habitudes déjà prises devaient, d'abord, être corrigées. Mais on se trouvait dans de bonnes conditions ; les spécialistes étaient encore peu nombreux et les relations entre eux étaient bonnes. Le travail mené en anglais et en français, et rapidement terminé, sert maintenant de modèle pour les autres langues, allemand, russe, japonais, etc.

Du côté des spécialistes, donc, tout avait bien marché, les revues spécialisées avaient diffusé la nouvelle terminologie. Le préfixe «cryo» devint à la mode pour la même raison que «fiable»: le prestige des nouvelles techniques. À la bourse de New York le cours des actions montait dès que «cryo » figurait dans la raison sociale. La publicité s'en mêlait bien entendu. Voulait-on, par exemple, vous vendre un produit à mélanger à l'essence de votre voiture, ou à l'huile du moteur ou à l'eau du radiateur, ce produit présenté dans les revues à grande diffusion par une ravissante personne, minijupée naturellement, était baptisé 《 cryogénique ».

Comment ne pas trouver désolant tout cela, qui risque de compromettre les efforts des savants et des ingénieurs ? Et pour quel bénéfice ? Or, cette manœuvre va se reproduire bien des fois, car sciences et techniques progresseront en élargissant leurs domaines, vers des pressions et des températures extrêmement hautes ou extrêmement basses, vers des degrés de pureté extrêmement élevés. Il y aura donc des acceptions nouvelles à nommer, et les termes choisis joueront des rôles de superlatifs. Par-là même, ils seront prestigieux. La publicité s'en emparera et les cycles ci-dessus décrits recommenceront.

Remarquons que ces superlatifs sont toujours relatifs. Les températures, par exemple, qui aujourd'hui nous paraissent extrêmement basses, seront un jour dépassées, et des industries se créeront pour les utiliser. Il faudra trouver une 
autre classe de préfixes. Les marques traditionnelles du superlatif ne peuvent pas servir longtemps. Il faut également prendre garde à ne pas bloquer la terminologie par des termes qui joueraient le rôle de superlatifs absolus, de bornes infranchissables. On achève actuellement un boulevard qui fait le tour de Paris; on l'appelle périphérique, alors qu'il est déjà noyé dans le tissu urbain. Comment nommera-t-on son successeur, qui ne sera pas moins périphérique, et que l'on prévoit dès maintenant vingt kilomètres au-delà ?

Maintenant que les timbaliers sont passés que reste-t-il ? Les mots honnêtes et simples, les mots humbles. Par définition on ne devrait pas en parler; ils font leur métier sans histoires. Ils le faisaient plutôt. Ils sont dévoyés. Par eux-mêmes innocents, on les force souvent à entrer en des assemblages prétentieux ou grotesques. Et il est salutaire de montrer que nous ne sommes pas dupes. Voici quelques échantillons : Le fabricant d'un chauffe-eau annonce qu'il a « réinventé l'eau chaude ». À un coureur qui gagne une course automobile, un journaliste demande : "Quand avez-vous senti que vous aviez la victoire au bout du volant? » (ce qui rejoint les «quatre coins de l'hexagone» de ministérielle et célèbre mémoire). Un publicitaire propose un produit de beauté qui fait « découvrir la vraie personnalité de votre peau ».

Un lexique du «marketing » donne du mot «univers » la définition suivante, dont on n'admirera jamais trop la rédaction : "ensemble de foyers que l'on étudie pour un problème donné au moyen d'un échantillon qui est représentatif. » Un exemple vient éclairer tout cela : "Ainsi l'univers des consommateurs de café en grains est composé de tous les français (sic) âgés de 8 ans et plus ». Accordez une pensée à ces pauvres petits enfants de 8 ans (et plus, il est vrai), qui n'ont pas encore fait de mal à personne, et qui doivent, cependant, concasser des grains de café avec leurs jolies petites dents !

Un autre auteur écrit un ouvrage sur la gestion des affaires; il utilise constamment le vocabulaire médical. Il est loin d'être le seul. Ce sont souvent des vocabulaires entiers qui sont ainsi sortis de leur domaine traditionnel. Le vocabulaire militaire est très demandé. Peut-être assouvit-on par là des désirs inconscients de puissance.

La médecine est à la fois un art et une science, tous deux difficiles à acquérir et à pratiquer. Quelle satisfaction que de s'identifier, ne serait-ce que par les mots, au médecin, "daïmon» redouté et souhaité! Diagnostic, pronostic sont employés si souvent que l'on commence à oublier - pour le second surtout qu'il s'agit de termes médicaux. Quant au mécanisme d'un attentat, pourquoi le démonter puisqu'on peut faire maintenant l'« autopsie » de l'attentat lui-même? Songez à la joie de la moindre charcutière quand elle déclare : « Mon mari est dans son laboratoire ».

Jamais le citoyen moyen n'a consommé autant de termes d'arithmétique ou de géométrie. Et l'archétype, le mot «problème », qui depuis l'enfance a laissé de mauvais souvenirs à d'autres qu'aux Delphine et Marinette de Marcel Aymé, on 
s'en venge en le mettant partout : «Si votre chien se gratte, c'est qu'il a des problèmes avec sa peau. »Voilà ce que l'on entend à la radio. Forme ridicule, et fond discutable, car ce n'est pas avec sa peau que la pauvre bête a des * problèmes », mais avec ce qui est à l'intérieur, et la lotion que cette publicité cherche à placer ne servira sans doute à rien, sauf à l'accroissement du chiffre d'affaires du vendeur.

A. Sauvageot, à juste titre, dénonce la terminologie «insensée » des mathématiques dites modernes. L'Académie des sciences de Paris vient d'être consultée par le père d'une petite fille de 9 ans à propos du mot «idempotent ». La maîtresse d'école avait parlé de « deux ensembles de ballons idempotents ». Le père n'avait trouvé le mot dans aucun dictionnaire. Plaise à l'Académie de dire s'il est indispensable qu'une enfant de cet âge sache un tel mot. Idempotent voulait dire qu'il y avait autant de ballons dans un tas que dans l'autre !

Peut-être faudrait-il frapper d'interdit le mot «moderne», mot de soufre pour les uns, mot de prestige pour d'autres. Il n'est pas très honnête d'abord. Si, en effet, par les temps qui courent, on parle beaucoup de ces mathématiques modernes, c'est uniquement en raison de leur imprudente introduction dans les enseignements primaire et secondaire. Car leurs éléments essentiels ont été imaginés au siècle dernier avec Galois (1830), avec Boole (milieu du siècle) et avec Cantor (son premier mémoire date de 1874). Le mot "moderne » devrait être proscrit également pour impropriété. Rien n'est jamais moderne. La modernité, comme l'actualité, est essentiellement éphémère. Ā peine née elle disparaît.

Avant ce délire verbal, les mathématiciens avaient pourtant bien pris la question. Ils résolvaient excellemment l'internationalisation de leur vocabulaire avec leur choix de mots humbles : boule, pavé, signature, qui ont, de par leur nature même, des correspondants dans les autres langues. Tout au plus pouvait-on leur signaler que l'application de leur science à un nombre croissant d'activités (biologie, économie, politique, finances, voire psychologie, etc.) s'accompagne d'une gêne croissante lorsque l'on doit user des termes généraux qu'ils ont particularisés comme : ensemble, groupe, suite, série...

Lorsqu'on se trouve dans le domaine d'une de ces sciences mathématisées, il n'est pas toujours facile de savoir si le mot (par exemple «groupe ») que l'on entend ou que l'on dit, a le sens du langage ordinaire ou l'acception mathématique précise.

Cette difficulté est accrue, dans certains cas, par l'évolution des théories mathématiques. Il y a aujourd'hui plusieurs théories des ensembles, et la première en date, celle qui a valu des ennuis à Cantor et ne lui a rapporté qu'une gloire surtout posthume, celle qui paraissait à ses contemporains scandaleuse et démoniaque, est maintenant qualifiée de «naïve». Le mot «ensemble » lui-même voit son champ rétréci ; son ancienne place a été donnée à un autre mot d'acception très générale : «collectif ».

On rencontre sous la plume de personnes qui ne sont pas des mathématiciens, des éloges du formalisme mathématique qui peut, disent-elles, se passer du langage 
ordinaire. Ce n'est vrai qu'en théorie. En pratique, il suffit d'ouvrir un ouvrage de mathématique, ou de logique, pour voir que le langage ordinaire est un ciment entre les placards de symboles. Un mathématicien piémontais, Joseph Peano, esprit d'une grande originalité, qui imagina des êtres mathématiques surprenants, a construit à la fin du dix-neuvième siècle un système de signes qui permet d'énoncer toutes les propositions de mathématique et de logique sans recours au langage ordinaire.

Or, personne ne s'est jamais servi du travail de Peano, les spécialistes jugeant trop grand l'effort qu'ils auraient dû faire pour assimiler cette langue particulière. Pourtant ils savaient que les langues ordinaires ont été à l'origine d'erreurs scientifiques ou philosophiques nombreuses et graves; ils auraient été récompensés de l'effort. Leur refus fait comprendre la négligence des gens instruits - mais non spécialistes - qui ne se donnent pas toujours la peine de veiller au sens des mots qu'ils emploient.

Les transferts de vocabulaires signalés plus haut ne facilitent pas le maintien du sens des mots tout le long d'un texte ou d'un exposé. Et lorsqu'on fait remarquer à ces gens instruits qu'ils déraillent, qu'ils passent d'une acception à l'autre, de l'acception spéciale à l'acception banale par exemple, ils expriment avec amertume leur souhait de monosémie : «Et voilà encore un mot qui a deux significations !» ou encore : "Cela n'arriverait pas si les mots n'avaient qu'un seul sens. » À leur décharge, il faut reconnaître que ce cap est difficile à tenir. En voici deux illustrations :

J'ai parlé de fiabilité, mais je n'ai pas donné de définition. Lorsqu'on en cherche une dans ce qui a été publié depuis quelques années, on est surpris par le grand nombre de définitions proposées. Certaines associations, désireuses de voir clair dans cette affaire, les ont collectionnées.

La fiabilité étant une qualité ne peut pas se mesurer directement; on en rattache les variations à celles d'une grandeur mesurable. Dans le cas présent cette grandeur est une probabilité. Le mot «probabilité », étant donné le sujet, a obligatoirement le sens précis qu'il a en mathématiques. La définition devrait donc comporter une description de la façon dont cette probabilité est mesurée. On constate que très généralement il n'en est rien. Les définitions que l'on rencontre sont floconneuses, inutilisables en pratique, le mot probabilité y est pris dans l'acception vague du langage courant (il est probable qu'il pleuvra demain). Ce sont, si l'on veut, des définitions pour gens du monde.

Nous avons vécu l'autre exemple au Comité d'étude des termes techniques français. L'A.F.N.O.R. nous avait demandé de l'aider à préciser la terminologie d'un chapitre de la mécanique. Nous nous sommes aperçus que, dans le projet que l'on nous proposait, les mots « choc », «percussion », « impulsion », étaient définis les uns par les autres, parce qu'ils étaient pris, à tort, dans leurs acceptions banales, qui sont peu différentes. Nous avions en face de nous un ingénieur de qualité, de bonne foi, désireux d'aboutir. Nous étions donc dans les meilleures conditions possibles, et malgré cela il nous a fallu un certain temps pour convaincre notre interlocuteur; ses objections reposaient sur les acceptions banales, alors 
que la nature du sujet imposait de les oublier et de se servir uniquement des acceptions de la mécanique rationnelle qui, elles, sont fort distinctes les unes des autres.

Les mots, les pauvres mots ont de grandes douleurs.

Leur sens ne changeait jadis que sous la pression de la nécessité. Ceux qui parlaient, qui écrivaient s'efforçaient de se faire comprendre. C'est là un scrupule qui se rencontre de plus en plus rarement. On a tendance aujourd'hui à laisser l'effort à celui qui lit ou qui écoute. Le résultat est une indifférence aux projets d'autrui et une ignorance que l'on ne soupçonne pas.

Pour que l'on en prenne conscience, il faut des enquêtes comme celles que conduit $\mathbf{M}^{\mathrm{me}} \mathrm{J}$. Ogée. Professeur à l'École de haut enseignement commercial pour jeunes filles (à Paris), elle a exposé à la biennale de Menton comment elle s'y prenait. Ses élèves, munies d'un questionnaire, vont prier les dirigeants parisiens du commerce, de l'industrie, les spécialistes du «marketing», du «merchandising $»$, etc., de leur donner des réponses. À priori il n'est pas possible d'imaginer la récolte de ces jeunes filles. Pourrait-on penser, par exemple, que sur 88 P.-D.G., 65 ignorent que le mot " royalty», que pourtant ils emploient constamment, a le sens de redevance? Les cadres supérieurs se comportent un peu mieux, mais tout de même, et pour garder le même exemple, $27 \%$ d'entre eux donnent à « royalty» un sens inexact ou refusent de répondre (ce qui est un aveu d'ignorance).

Le remède n'est pas dans la recherche d'une monosémie, qui serait impossible à réaliser même en sciences. Les mots se chargeraient vite d'affectivité, et les sens glisseraient. Le plus efficace, de ce point de vue, serait de ne laisser dans le vocabulaire que des mots de soufre, c'est-à-dire de rendre la vie infernale, à la manière des personnages du roman de Georges Orwell. Il est permis de ne pas retenir pareille solution.

S'il ne faut pas manquer une occasion d'agir sur la langue, de la simplifier quand cela est possible, de la compléter, le remède n'est pas dans une action sur l'outil, mais sur l'utilisateur de l'outil. S'il faut donner à cet utilisateur une langue aussi bien faite que possible, cela n'est pas suffisant. «C'est le besoin qui crée la conscience », dit Jean Piaget. Répétons qu'il faut, avec patience, montrer aux gens qu'ils ont besoin d'une langue précise. Mais cette action manquera d'acuité tant que nous n'aurons pas étudié la psychologie de ces « locuteurs 》 tant actifs (journalistes, hommes de la radio, de la télévision, publicitaires) que passifs. Comment réagit chacun d'eux lorsqu'il entend des métaphores ridicules ou du charabia prétentieux, lorsqu'on lui propose des mots nouveaux. Pourquoi acceptet-il d'emblée certains de ces mots ; pourquoi en refuse-t-il d'autres?

Tout cela sans nous dissimuler que la dégradation des mœurs rend précaire l'effet de nos tentatives. Il faut pour persévérer songer à l'un de ces personnages symboliques que la littérature, comme la physique tout à l'heure, ne dédaigne pas : le passeur au roseau vert qui rame à contre-courant.

Pierre Agron 